\title{
ROLE OF DAIGNOSTIC ULTRASONOGRAPHY IN EVALUATION OF HAND ANDWRIST LESIONS
}

\author{
Osama A. AllaDawoud, Ibraheem A. Lebida, Dalia Nabil Khalel and Mahmoud M.Gabal \\ (Radiodiagnosisdepartments, Faculty of Medicine, ZagazigUniversity).
}

\begin{abstract}
Objective: To highlight the role of high resolution US (ultra sound) in diagnosis of hand and wrist lesions. Materials And Methods: This study was performed at Radiology department, Zagazig university hospitals during the period from January 2011 to December 2015 .one hundred patients complaining of wrist or hand problem and fifteen normal volunteers subjects as control group were enrolled in the study and uponsuspected clinical diagnosisthey wear grouped into five groups. High resolution US was done for all cases and control subjects. Results following surgical evaluation or clinical and radiological follow up were compared with the US results. Sensitivity, specificity, PPV, NPV and accuracy were calculated.

Results:Group A included 40 cases:Carpal tunnel syndrome (CTS) was detected in 20 patient, traumatic or post-operative nerve injury was presented in 20 cases, Majority of cases 10 cases (50\%) showed complete chronic nerve cut with neuroma in continuity. Group B included 35 cases $40 \%$ of the patients had complete tendon cut, $17.2 \%$ had partial thickness tendon cut, $14.3 \%$ isolated tenosynovitis and $28.6 \%$ had tendon entrapment. Group C included 10 cases with arthritis; Group D included 8 cases with hand or wrist mass, and group E included 7 cases with foreign body impaction. The sensitivity, specificity, PPV, NPV and accuracy of US examination in diagnosing different wrist and hand pathology in those groups were high reaching up to $100 \%$ in most of these pathologies exceptfor complete traumatic tendon cut the sensitivity, specificity, PPV, NPV and accuracy of ultrasound were $87.5 \%, 100 \%, 100 \%, 75 \%, 90 \%$,for partial tendon cut were $100 \%, 87.5 \%, 66.7 \%, 100 \%, 90 \%$, for the complete nerve injury were $100 \%, 90.9 \%, 90 \% 100 \%, 95 \%$ and for partial nerve injury were $77.8 \%, 100 \%, 100 \%, 84.6 \%, 90 \%$ respectively.

Conclusions:Us has high sensitivity and specificity in detecting most of the hand and wrist pathology. It can be used for pre-operative planning.It is low cost and Lacking of ionizing radiation made it the first imaging modality for hand and wrist lesions.
\end{abstract}

Keywords: High resolution US, hand and wrist lesions, CTS, nerve injury

\begin{tabular}{lc}
\hline Corresponding Author: & Received: February 2016 \\
Name: Mahmoud Gabal & \\
Phone numbers: +20010105137897 & Accepted: April 2016 \\
\hline
\end{tabular}

\section{INTRODUCTION}

M usculoskeletal ultrasonography (US) of the hands and wrist recently has been increasing in popularity. Rapid technical advances in the US, such as new ultra-high frequency probes and smaller probe sizes, have led to improved image quality ${ }^{(1)}$.

This, in turn, has accelerated the growth of musculoskeletal US. Superficial structures of the hands and wrist, including the tendons, ligaments, nerves are amenable to imaging with high frequency US. Known US advantages are lack of ionizing radiation, noninvasiveness, portability and low cost ${ }^{(\mathbf{1})}$.

Sonography is an excellent modality for investigating many structures of the hand and wrist. It is best used when the problem is well localized and when the clinical question is relatively specific ${ }^{(2)}$.
US can assess the continuity and integrity of the nerve, characterize the defect, and identify secondary nerve compression. Thereby, location, extent and type of damage were determined. This allows displaying a complete and partial nerve transection, the distance and condition of the stumps (formation of a neuroma) or a compression of the nerve, for example by scars, ostheosynthetic material, callus formation, bone fragments, hematomas, or foreign bodies $^{(3)}$.

Ultrasonography can demonstrate the intrinsic changes affecting the median nerve including its cross section area, flatting ratio, mobility and any anatomical variants. Ultrasonography can also demonstrate the extrinsic abnormalities at the carpal tunnel as, tendon pathology, ganglions and aberrant muscles ${ }^{(4)}$. 
Tenosynovitis appears on sonography as thickened hypoechoic tissue with or without fluid within the tendon sheath ${ }^{(5)}$.

The reflectivity of a foreign body depends on its acoustic impedance on US ${ }^{(\mathbf{6})}$.

The aim of this study was to highlight the role of high resolution US (ultra sound) in diagnosis of hand and wrist lesions.

\section{PATIENTS AND METHODS}

This study included 100 patients with wrist or hand lesion of different causes. They were 48 females (48\%) and 52 males (52\%). Their age ranged from 1 to 70 years and mean age was $33.4 \pm 8.7$ year. The study was performed during the period from January 2011 to December 2015.All our cases were referred from rheumatology andOrthopedics outpatient clinics of Zagazig university Hospitals.

Approval for the study from ethics committee board of our institute was taken, as well as, a written consent from all participants after explanation of the procedure.

No need for sedation during children examination as our study was non invasive nor painful.

The patients were subjected to the following:

1.Clinical history taking

2. Clinical examination

3. Imaging modalities:

a-Plain X ray for some selected cases

b-High resolution US:

Technique of US:

Siemens SonolineAdara ultrasound imaging system using $10 \mathrm{MHZ}$ linear probe was used.

\section{Technique of carpal tunnel examination:}

- The probe was placed at the level of the distal skin crease.

- In the axial plane, the ulnar artery is easily located medially

- The sonographic beam needs to be perpendicular to the surface of the flexor tendons. Just with identification of the articulation with the scaphoid more laterally or the lunate medially we shift the probe to the transverse direction and the carpal tunnel is seen at its beginning.

- The flexor tendons examination started from the wrist joint and continued through the whole course of the tendons of interest.
- Quantitative measurements of the cross sectional area of the tendon was done with the comparison with the healthy tendon on the other hand

- The median nerve size, shape, echogenicity and relationship to the underlying tendons and the overlying retinaculum were noted.

\section{Technique for Nerve injury examination}

- We started examination from proximal distance of the area of suspected injury. Axial and longitudinal images were recorded on US printing papers,

- The ratio of the CSA (cross sectional area) at the forearm to the CSA of the nerve at the wrist should be 1 .

- We direct trace the median nerve as a self-control of the case normally the ratio is less than 1.3.

Examination of the flexor apparatus of the hand:

- The flexor tendons examination started from the wrist joint and continued through the whole course of the tendons of interest to the insertion point with special concentration on synovial sheaths, areas of bifurcation and reunion of FS tendons, and the flexor belly system.

Technique of the examination of the extensor apparatus:

- The bony land marks were the key structures that define the sonographic anatomy of the extensor surface of the wrist, dorsal tubercle of the radius and the radioulnar joint.

\section{Technique for Small joint examination:}

- Both articular cartilages on dorsal and ventral sides of the proximal articular aspect are evaluated

- The synovial recesses were evaluated the degree of thickening was evaluated regarding the slandered measurements in relation to the head of the metacarpal bone.

\section{Technique of Soft tissue masses examination:}

Soft tissue masses examination started by trail to characterize the mass as a soft tissue or bony in texture. With soft masses we tried to know the nature of the mass as a fluid or soft tissue. 


\section{Surgical evaluation:}

Surgery was performed in 58 cases: 20 cases for CTS, 10 patients with nerve injury, 17 cases with tendon trauma, 3 cases for tendon sheath removal due to tenosynovitis and 8 cases with localized mass.

\section{Conservative management and follow up:}

Conservative management in the form of medical treatment and follow up was done in the other 42 cases.

\section{Statistical analysis of data:}

Validity of (US) for diagnosis hand and wrist problems was tested by sensitivity, specificity, positive predictive value (PPV) and negative predictive value $(\mathrm{NPV}) \&$ $\operatorname{accuracy}^{(7)}$.

\section{RESULTS}

This study included 100 patients with wrist and hand lesions of different causes and fifteen normal volunteer subjects as control group. They were 48 females (48\%) and 52 males $(52 \%)$. Their age ranged from 1 to 70 years and mean age was $33.4 \pm 8.7$ year. The most common affected age group in this study was between 30-40 years found in 30\% of the patients. The most common clinical presentation in our patients was swelling in hand or wrist presented in $65 \%$ of the patients

\section{According to suspected clinical diagnosis our 100 patients were classified into five groups:}

> Group A included 40 cases (table 1):Carpal tunnel syndrome (CTS) was detected in 20 patients. The most common detected sign in these cases was increased size of the median nerve which was detected in all cases with idiopathic CTS (10cases) and 4 cases with non idiopathic CTs, followed by flattening of the median nerve ( 5 cases), bowing of flexor retinaculum ( 5 cases). Regarding the 10 cases with non idiopathic CTS, four patients had past history of CTS release and showed Enlarged swollen nerve (recurrent CTS). Two cases show median nerve variants in the form of bifid median nerve. Two cases showed Persistent median artery, aberrant lumbrical muscle (one case) and deep ganglion (one case).Traumatic or post-operative nerve injury was presented in 20 cases(table 2), involving median nerve in $18(90 \%)$ cases and ulnar nerve $2(10 \%)$ cases. the most frequently affected site for median and ulnar nerves cut in our patients was at the wrist presented in 17 patients $(\mathbf{8 5 \%})$ Majority of cases 10 cases (50\%) showed complete chronic nerve cut with neuroma in continuity, 7 cases had partial nerve cut and 3 cases had neuropathy.

Group B included 35 cases(table 3) $40 \%$ of the patients had complete tendon cut, $17.2 \%$ had partial thickness tendon cut, $14.3 \%$ isolated tenosynovitis and $28.6 \%$ had tendon entrapment .Two out of our 20 patients diagnosed by ultrasound as partial tendon injury show complete tendon injury during surgery. We reported 15 cases with tenosynovitis, 10 cases with tendon entrapment due to tenosynovitis, and 5 cases had isolated tenosynovitis

$>$ Group C included 10 cases with arthritis (table 4). The most significant sign in arthritis was bone erosion found in 8 cases $(80 \%)$ associated with synovial thickening (7 cases) and effusion ( 7 cases). Most of the cases had 2 to 3 finding together.

$>$ Group D included 8 cases with hand or wrist mass. 4 cases with ganglion in between the extensor tendons on the dorsum of the hand, 2 cases in the ventral aspect of the hand and 2 cases FDP tendon sheath at its insertion in the middle finger. The final ultrasound diagnosis of the mass lesions was 6 cases of ganglion and two cases of GCT.

$>$ Group E included 7 cases with foreign body impaction.

$>$ The sensitivity, specificity, PPV, NPV and accuracy of US examination (table 5) in diagnosing different wrist and hand pathology in those groups were high reaching up to $100 \%$ in most of these pathologies except for complete traumatic tendon cut the sensitivity, specificity, PPV, NPV and accuracy of ultrasound were $87.5 \%, 100 \%, 100 \%, 75 \%$, $90 \%$, for partial tendon cut were $100 \%$, $87.5 \%, 66.7 \%, 100 \%, 90 \%$, for the complete nerve injury were $100 \%, 90.9 \%, 90 \% 100 \%$, $95 \%$ and for partial nerve injury were 77.8 $\%, 100 \%, 100 \%, 84.6 \%, 90 \%$ respectively. 
Table (1): Shows type of nerve insult as diagnosed by US for 40 patients.

\begin{tabular}{lcc}
\hline US diagnosis & No & \% \\
\hline Idiopathic CTS & 10 & 25 \\
\hline Non idiopathic- CTS & 10 & 25 \\
\hline Median nerve injury & 18 & 45 \\
\hline Ulnar nerve injury & 2 & 5 \\
\hline Total & 40 & 100 \\
\hline
\end{tabular}

Table (2): US findings in 20 patients presented with median and ulnar nerves injury in this study.

\begin{tabular}{lccl}
\hline \multicolumn{1}{c}{ US finding } & No & $\%$ & US diagnosis \\
\hline $\begin{array}{l}\text { Gap with terminal neuroma of the median } \\
\text { nerve }\end{array}$ & 10 & 50 & Complete post traumatic nerve cut \\
\hline $\begin{array}{l}\text { Intact sheath with partial loss of fascicle of } \\
\text { the median nerve }\end{array}$ & 3 & 15 & Partial post traumatic nerve cut \\
\hline Sheath loss with no scar entrapment & 2 & 10 & Partial post traumatic nerve cut \\
\hline Sheath loss with entrapment by scar & 2 & 10 & Partial post traumatic nerve cut \\
\hline $\begin{array}{l}\text { Intact sheath with loss of fascicle of the } \\
\text { median nerve }\end{array}$ & 3 & 15 & Post traumatic neuropathy \\
\hline
\end{tabular}

Table(3): shows US finding in 35 cases suspected to have tendon insult

\begin{tabular}{|c|c|c|c|}
\hline US finding & No & $\%$ & US diagnosis \\
\hline Interrupted course with retraction & 14 & 40 & Complete tendon cut \\
\hline $\begin{array}{l}\text { Swollen tendon with hypoechoic foci and } \\
\text { effusion }\end{array}$ & 6 & 17.2 & Partial thickness tendon cut \\
\hline $\begin{array}{l}\text { Fluid distending the tendon sheath and/or } \\
\text { thickening of the tendon sheath }\end{array}$ & 5 & 14.3 & Isolated tenosynovitis \\
\hline Thickening of the tendon sheath & 10 & 28.6 & Tendon entrapment \\
\hline Total & 35 & 100 & \\
\hline
\end{tabular}

Table (4): shows Ultrasound Findings in 10 cases with arthritis

\begin{tabular}{ccc}
\hline Finding & Number & $\%$ \\
\hline Effusion & 7 & 70 \\
\hline Synovial thickening & 7 & 70 \\
\hline Bone erosion & 8 & 80 \\
\hline
\end{tabular}


Table (5):the ultrasound diagnosis and final diagnosis in the 100 included patients

\begin{tabular}{|c|c|c|c|c|c|c|c|c|c|}
\hline & \multicolumn{2}{|c|}{ US diagnosis } & \multicolumn{2}{|c|}{$\begin{array}{c}\text { Final } \\
\text { diagnosis }\end{array}$} & \multirow[t]{2}{*}{ Sens. } & \multirow[t]{2}{*}{ Spec. } & \multirow[t]{2}{*}{ PPV } & \multirow[t]{2}{*}{ NPV } & \multirow[t]{2}{*}{ Accuracy } \\
\hline & No & $\%$ & No & $\%$ & & & & & \\
\hline \multicolumn{10}{|l|}{ Nerve disorders } \\
\hline Entrapment & 20 & 50 & 20 & 50 & 100 & 100 & 100 & 100 & 100 \\
\hline \multicolumn{10}{|l|}{ Trauma } \\
\hline Complete cut & 10 & 25 & 9 & 20 & 100 & 90.9 & 90 & 100 & 95 \\
\hline Partial cut & 7 & 17.5 & 9 & 22.5 & 77.8 & 100 & 100 & 84.6 & 90 \\
\hline Neuropathy & 3 & 7.5 & 2 & 7.5 & 100 & 94 & 66.7 & 100 & 95 \\
\hline \multicolumn{10}{|l|}{ Tendon disorders } \\
\hline Tenosynovitis & 5 & 14 & 5 & 14 & 100 & 100 & 100 & 100 & 100 \\
\hline Entrapment & 10 & 29 & 10 & 29 & 100 & 100 & 100 & 100 & 100 \\
\hline \multicolumn{10}{|l|}{ Trauma } \\
\hline Complete cut & 14 & 40 & 16 & 46 & 87.5 & 100 & 100 & 75 & 90 \\
\hline Partial cut & 6 & 17 & 4 & 11 & 100 & 87.5 & 66.7 & 100 & 90 \\
\hline \multicolumn{10}{|l|}{ Masses } \\
\hline Ganglion & 6 & 80 & 6 & 80 & 100 & 100 & 100 & 100 & 100 \\
\hline GCT of tendon sheath & 2 & 20 & 2 & 20 & 100 & 100 & 100 & 100 & 100 \\
\hline \multicolumn{10}{|l|}{$\underline{\text { Arthritis }}$} \\
\hline Rheumatoid arthritis & 9 & 90 & 9 & 90 & 100 & 100 & 100 & 100 & 100 \\
\hline Psoriasis & 1 & 10 & 1 & 10 & 100 & 100 & 100 & 100 & 100 \\
\hline Foreign body & 7 & 100 & 7 & 100 & 100 & 100 & 100 & 100 & 100 \\
\hline
\end{tabular}

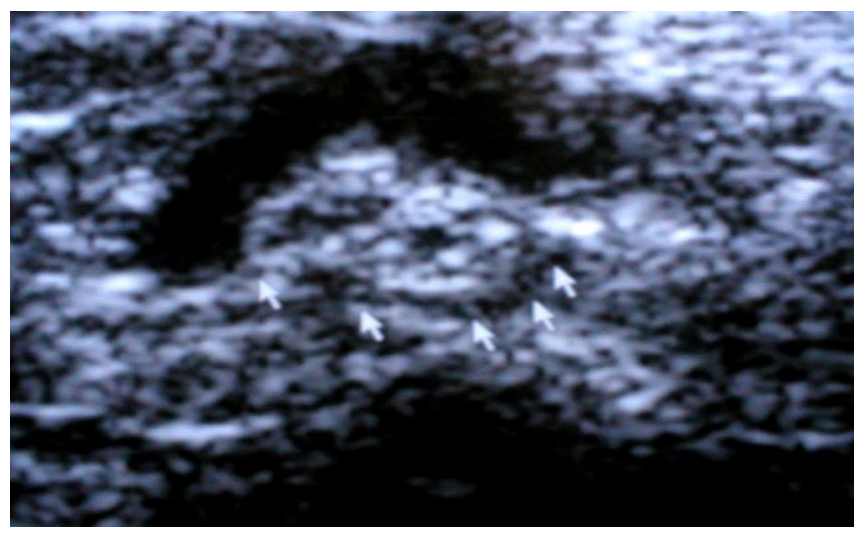

(A)

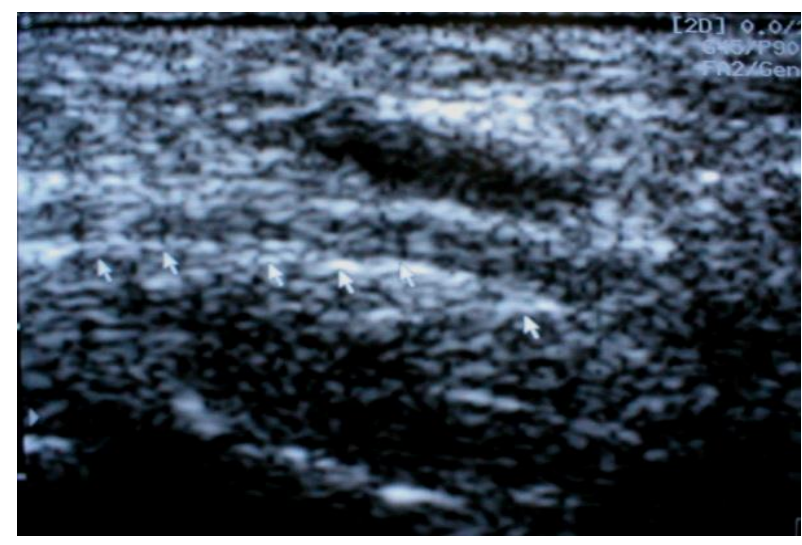

(B)

Case No.1: post traumatic scar tissue entrapment of the left median nerve.Figure [A] transverse high resolution ultrasound scan at the wrist show hypoechoic scar tissue superficial to the left median nerve (white arrows) and forming an arch around its ventral aspect. Figure [B] longitudinal cut shows long scar sheath ventral to the median nerve (white arrows). 


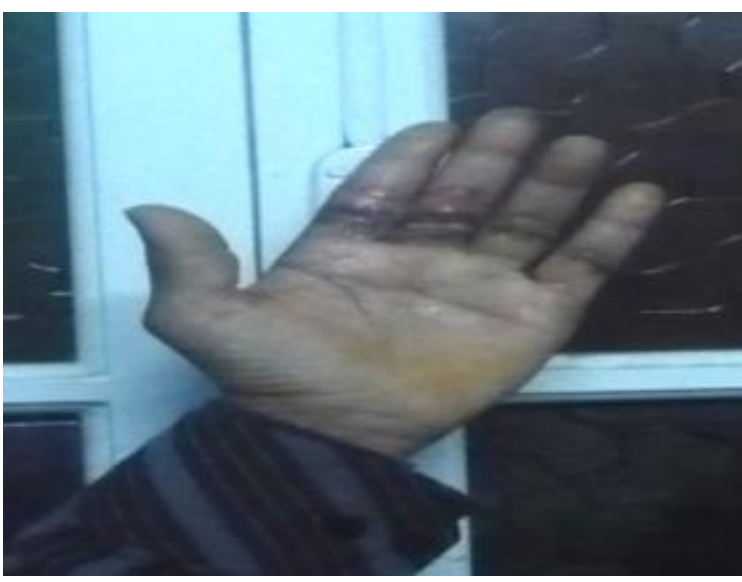

(A)

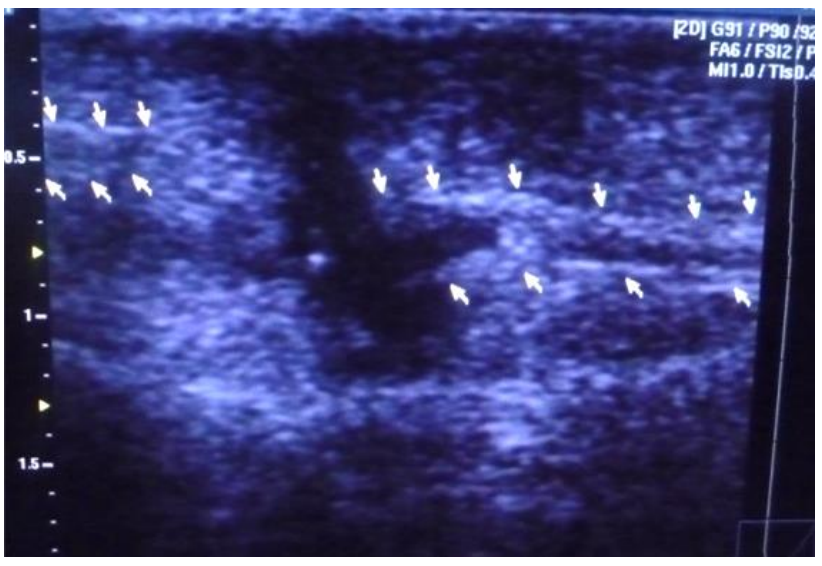

(B)

Case No.2: complete tear of the digital branch of the median nerve of the left index finger. Figure [A] photograph of the left hand shows cut injury to the proximal part of the ventral aspect of the left four fingers by a knife.Figure [B] Longitudinal high resolution sonogram shows complete cut and separation of the digital branch of the median nerve of the index finger [arrows] with extensive scar tissue between the two ends of the nerve and extend to bone,

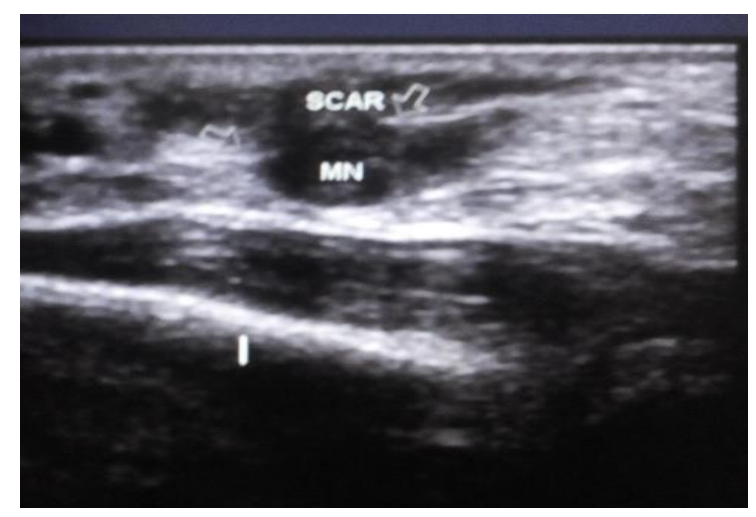

(A)

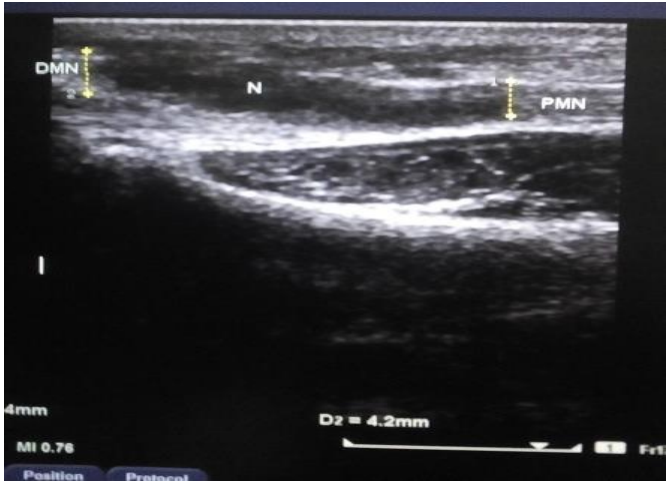

(B)

Case no. 3: neuroma in continuity.Figure [A] short axis high resolution sonograph show loss of normal fascicular pattern of the right median nerve with loss of ventral sheath and the nerve is entrapped by scar tissue.Figure [B] long axis high resolution sonograph shows the median nerve with loss its normal fascicular pattern

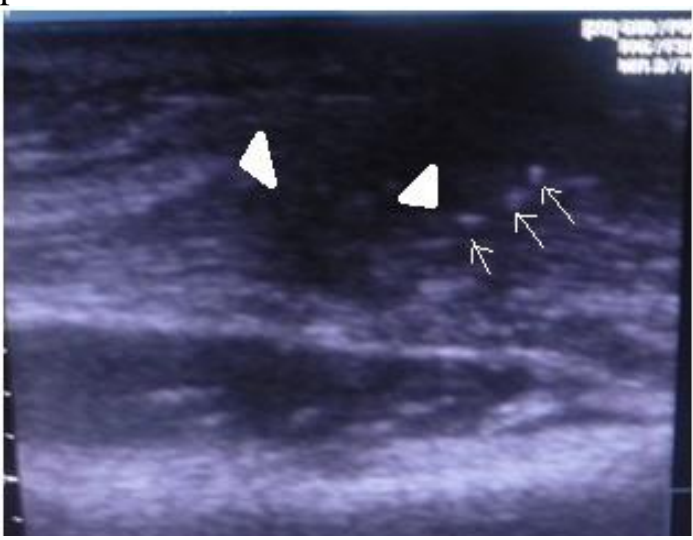

(A)

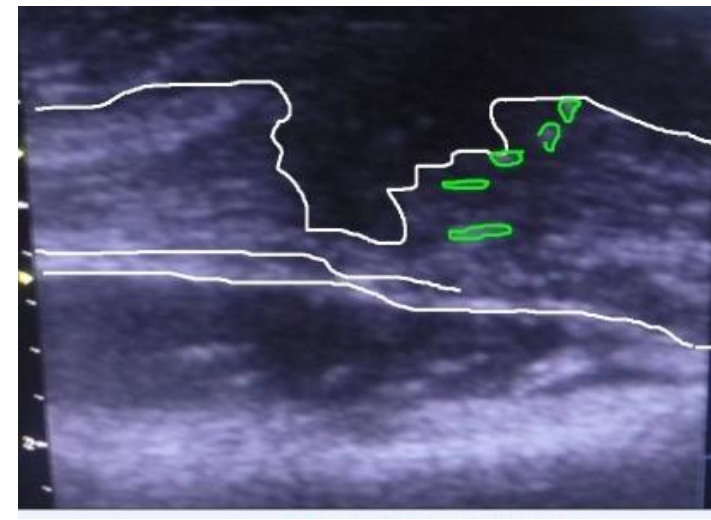

(B)

Case no 4: post surgical repair suture dehiscence of FPL tendonFigure [A] Longitudinal high resolution sonogram shows loss of ventral continuity of the FPL tendon with hypoechoic scar tissue filling the gap between the separated end of the tendon (arrow head) and suture dehiscence (small arrows). Figure [B] illustrated diagram of figure $\mathrm{A}$; the tendon is outlined in white the suture in green. 


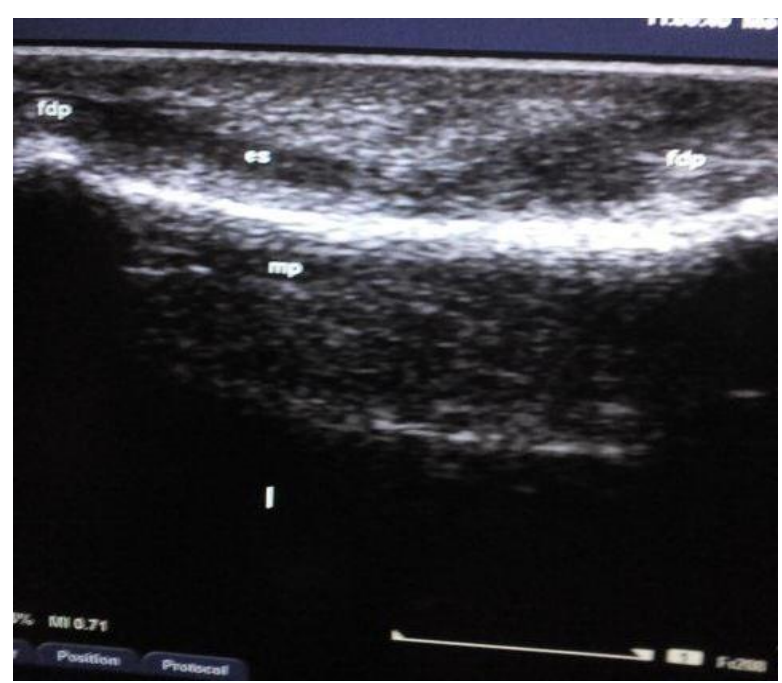

(A)

Case No 5: partial tear of the flexor digitourm profundus tendon Figure [A] Long axis high resolution sonogram show loss of normal fibillar pattern of the flexor digitourm profundus tendon with some preserved fibers.

\section{DISCUSSION}

Musculoskeletal ultrasonography (US) of the hands and wrist recently has been increasing in popularity. Rapid technical advances in the US, such as new ultra-high frequency probes and smaller probe sizes, have led to improved image quality ${ }^{(1)}$.

This, in turn, has accelerated the growth of musculoskeletal US. Superficial structures of the hands and wrist, including the tendons, ligaments, nerves are amenable to imaging with high frequency US. Known US advantages are lack of ionizing radiation, noninvasiveness, portability and low cost ${ }^{(\mathbf{1})}$.

This study included 100 patients with wrist or hand lesion of different causes. They were 48 females (48\%) and 52 males $(52 \%)$. Their age ranged from 1 to 70 years and mean age was 33.4 \pm 8.7 year. The most affected age group in our study was between $30-40$ years (30\%). The most common clinical presentation in our patients was swelling in hand or wrist presented in 65 patients $(65 \%)$.

According to suspected clinical diagnosis our 100 patients were classified into five groups:

A. Patients with suspected nerve lesions (40 cases)

B. Patients with suspected tendon lesions ( 35 cases)

C. Patient with mass lesion (8 cases)

D. Patient with arthritis ( 10 cases)
E. Patient with foreign body impaction (7 cases).

The most affected group in our patients was patients with suspected nerve lesion presented in 40 patients (40\%). US can assess the continuity and integrity of the nerve, characterize the defect, and identify secondary nerve compression. Thereby, location, extent and type of damage were determined. This allows displaying a complete and partial nerve transection, the distance and condition of the stumps (formation of a neuroma) or a compression of the nerve, for example by scars, ostheosynthetic material, callus formation, bone fragments, hematomas, or foreign bodies $^{(3)}$.

Different cut off points of the cross section area of the median nerve swelling at CTS were used ${ }^{(\mathbf{8})}$ used $10.5 \mathrm{~mm}^{2}$ just proximal to the flexor retinaculum as the most useful ultrasound sign in making a positive diagnosis of CTS. Meanwhile, Sernik. ${ }^{(9)}$ considered a cut off point $10 \mathrm{~mm}^{2}$ more accurate for diagnosing CTS.

In agreement with Sarraf $\boldsymbol{e t} \boldsymbol{a l}^{(\mathbf{8})}$ we found median nerve swallowing in 18 cases using cross sectional area of $10.5 \mathrm{~mm}^{2}$ is the most diagnostic cut off point

When the nerve is compressed in the mid and distal tunnel, it flattens with an increased width-to-height ratio. It was suggested to be 
associated with CTS (case No.1) if this ratio is $3: 1$ or more ${ }^{(\mathbf{1 0})}$.

This is in agreement with our results as we found median nerve flattening in 5 cases with width-to-height ratio 3.4.

Cokluk. ${ }^{(11)}$ used High-resolution ultrasonography successfully to evaluate 14 cases of traumatic peripheral nerve injury preoperatively(case No.2). It revealed axonal swelling, neuroma formation, complete nerve transaction prior to surgery and improvement of subsequent surgical planning.

Kele $^{(3)}$, described the Stump or amputation neuromas by using high resolution ultrasonography as focal thickening or mass-like lesions at the nerve end, moreover, Cokluk. and Aydin. ${ }^{(11)}$ aided the clinical application of the high resolution ultrasonography by its benefits and feasibility in presurgical and intraoperative ultrasonographic examination of the upper and lower extremities in the localization and description of injury, evaluation of nerve stumps, diagnosing a stump neuroma, determination of perilesional scar formation, detecting foreign missiles in and around the injury site and in assessing the severity of the lesion.

This is in agreement with our results as 20 patients with history of trauma (cut injury with glass, stab wounds sharp machines) or pervious surgery [e.g. CTS release or nerve repair ] were examined for assessment of nerve injury, Nine of 10 cases with complete chronic nerve cut showed complete discontinuity of the fibers and neuroma formation (case No.3)on surgical management, however the other case showed partial interruption on exploration, while another one case diagnosed as neuropathy showed residual disability on follow up and considered as partial tear. 7 cases showed partial nerve cut from them (4 cases) showed sheath loss with scar entrapment ( 2 cases) and 2 cases without scar entrapment (2 cases). However 3 cases showed Intact sheath with partial loss of fascicle of the median nerve. In our study 3 cases showed no loss of continuity but loss of normal fascicular pattern and enlarged swollen appearance of the nerve trunk denoting neuropathy without interruption and diagnosed as neuropathy,
One of them show partial nerve cut on follow up.

Cartwright et al. ${ }^{(12)}$, stated that Highresolution ultrasound was able to identify transected nerves in the upper extremity with $89 \%$ sensitivity and $95 \%$ specificity. In agreement with Cartwright et al. ${ }^{(\mathbf{1 2})}$, our study found that that high resolution US hade sensitivity, specificity, PPV,PV and accuracy of $100 \%, 90.9 \%, 90 \% 100 \%, 95 \%$ and $77.8 \%, 100 \%, 100 \%, 84.6 \%, 90 \%$ and $100 \%, 94 \%, 66.7 \%, 100 \%, 95 \%$ for complete, partial nerve cut and neuropathy respectively.

We examined 35 case referred for different tendon pathologies, 20 with history of trauma, 10 with stenosing tenosynovitis \{either De.Qurvian syndrome or trigger finger $\}$ and 5 cases as suspected tenosynovitis.

In agreement with Azócar ${ }^{(13)}$, twenty cases with history of recent trauma, 14 cases with complete cut showed, discontinuity with retraction of cuted ends, however, gap filled with fluid was detected in only 6 cases. Six cases with partial cut, showed increase in the cross sectional area of the tendon and only hypoechoic foci in cross section with still intact fibers. On surgical management all the 14 patients demonstrated complete tendon cut, more over another 2 cases which were diagnosed by US as partial cut revealed complete transection of their fibers.

Our result is similar to Wang et al. ${ }^{(14)}$ who used ultrasonic assistance in the diagnosis of flexor tendon injuries and showed that USG was used to make correct preoperative determinations in six of the eight patients they studied(case No.4 \& 5).

Also we agree with Jeyapalan et al. ${ }^{(15)}$, who used USG to establish tendon pathologies in 17 patients in 18 digits. Surgery was undertaken in only three cases; two of these surgical cases confirmed USG findings. USG imaging helped to avoid surgery in 14 cases by excluding flexor tendon ruptures.

In agreement with Jeyapalan et al. ${ }^{(15)}$, our result had sensitivity, specificity, PPV, NPV and accuracy of $87.5 \%, 100 \%, 100 \%$, $75 \%, 90 \%$ and $100 \%, 87.5 \%, 66.7 \%, 100 \%$, $90 \%$ for complete and partial traumatic tendon cut respectively. 
McAlinden and $\mathbf{T e h}^{(5)}$ reported that, Tenosynovitis appears on sonography as thickened hypoechoic or anechoic tissue with or without fluid within the tendon sheath seen in two perpendicular planes, The tendon itself may be thickened, enlarged, and poorly defined with reduced echogenicity. In agreement with them, we were able to detect obvious signs for tenosynovitis and differentiate cases with entrapment. From 15 cases with tendon sheath thickening and effusion 10 of them were described as tendon entrapment due to specific location, 6 of them at the level of the metacarpal head entrapped by thickened first annular pulley (triggering) and 4 cases at the radial styloid process by thickened first extensor compartment sheath(De.Qurvian). 7 cases were treated by local injection of corticosteroid and the other 3 cases were operated for tendon sheath removal.

Ganglion cysts are the most common cause for palpable masses in the hand and wrist. They are most common in young women, although they can occur at any age and in both sexes ${ }^{(16)}$. In agreement with Angelides. ${ }^{(16)}$ we detect 6 ganglions in our 8 cases presented with hand and wrist swelling, 4 of them in young women [66\% of the ganglion cases.

In agreement with Angelides. ${ }^{(16)}$ we found that, the dorsal ganglion is the majority between our cases the origin from the scaphulonate ligament was detected in 3 of our 4 cases and the pedicle could be traced to the ganglion.

Greendyke et al. ${ }^{(17)}$ found Thirteen to twenty percent of ganglia on the volar aspect of the wrist, arising via a pedicle from the radio scaphoid/scapholunate interval, scaphotrapezial joint, or the metacarpotrapezial joint, in that order of frequency, and in agreement with Greendyke et al. ${ }^{(17)}$ we found 2 out of 6 ganglion cases originated from the volar aspect of the wrist and one pedicle could be traced to its origin from joint.

Middleton et al. ${ }^{(18)}$ stated that giant cell tumors of the hand typically appear as solid, homogeneous hypoechoic masses with detectable internal vascularity that are associated with the flexor tendons of the fingers. In agree withMiddleton et al. ${ }^{(\mathbf{1 8})}$, we found 2 of our cases complaining from mass lesion seen arising from flexor tendon of the fingers with hypoechogenisy and well defined capsule to be giant cell tumors after surgery and histopathology examination.

In the hand, rheumatoid arthritis is characterized by synovial proliferation, which can progress to bone erosions, tendon rupture, joint destruction, and deformity ${ }^{(\mathbf{1 9})}$.

In our study we evaluate 9 cases with reheumatoid artheritis and one case with arthritis due to psoriasis we found bone erosion in 8 cases $(80 \%)$ associated with synovial thickening( 7 cases $)$ and effusion (7 cases), most of the cases has 2 to 3 finding together. No evidence of bone destruction or tendon rupture was detected by US in our cases as all our patient were in early stages

Blankstein et al. ${ }^{(6)}$ reported that, Foreign bodies appear as hyperechoic structures with acoustic shadowing; a comet tail artifact can be seen if metallic fragments exist. The reflectivity of a foreign body depends on its acoustic impedance on US. This is agree with our study as we found 7 cases with chronic foreign body impaction. In all cases The FB appeared hyperechoic by US and surrounded by hypoechoic halo of granulation tissue and edema.

The limitation of our study was ignoring the role of MRI as a diagnostic modality for soft tissue lesions. Also US is operator dependent. So we recommend further studies for comparing sensitivity and specificity of MRI and high resolution US.

This stud had limitation first is the small number of patients as in soft tissue masses and arthritis groups, second we don't have patients with early rheumatoid arthritis.

Conclusion: In our study we found that high resolution US had $100 \%$ sensitivity in diagnosis of most of studied hand and wrist lesions, a part from partial nerve cut and complete tendon cut and had $100 \%$ specificity in diagnosis of most of studied hand and wrist lesions, a part from complete nerve cut and partial tendon cut. US was easy, safe and costless diagnostic imaging modality and can be used as preliminary method to detect wrist and hand lesions for early diagnosis and pre-operative evaluation. 


\section{REFERENCES}

1- Wong DC, Wansaicheong GK, Tsou IY. Ultrasonography of the hand and wrist. Singapore Med J. 2009; 50:219-225, quiz 226.

2- Middleton WD, Teefey SA, Boyer MI. Hand and Wrist Sonography. Ultrasound Quarterly. 2001; 17: 21-36.

3- Kele H. Ultrasonography of the peripheral nervous system. Perspect Med. 2012;1:417421.

4- Martinoli C, Bianchi S, Gandolfo N, Valle M, Simonetti S, Derchi LE. US of nerve entrapments in osteofibrous tunnels of the upper and lower limbs. Radiographics. 2000; 20: 213-217.

5- McAlinden $\mathbf{P}$, Teh $\mathbf{J}$. Imaging of the wrist. Imaging. 2003; 15:180-192.

6- Blankstein A, Cohen I, Heiman Z, et al. Localization, detection, and guided removal of soft tissue in the hand using sonography. Arch Orthop Trauma Surg. 2000; 120: 514.

7- Norusis M. Statistical package for social science (SPSS) base 10 for windows. user's guide. Chichago,IL:SPSS; 1997.

8- Sarraf P, Malek M, Ghajarzadeh M, Miri S, Parhizgar E, Emami-Razavi SZ. The best cutoff point for median nerve cross sectional area at the level of carpal tunnel inlet. Acta MedicaIranica. 2014; 52: 613-618.

9- Sernik RA, Abicalaf CA, Pimentel BF, Braga-Baiak A, Braga $L$, et al. Ultrasound features of carpal tunnel syndrome: a prospective case-control study. Skeletal Radiol. 2008 ;37: 49-53.

10- Chen P, Maklad N, et al. Dynamic highresolution sonography of the carpal tunnel. AJR Am J Roentgenol. 1997; vol 168: 533-7.
11- Cokluk C, Aydin K. Ultrasound examination in the surgical treatment of lower extremity peripheral nerve injuries: part II. Turk Neurosurg. 2007; 17:197-201

12- Cartwright MS, Shin HW, Walker FO. Ultrasonographic characteristics of the normal median nerve. Neurology. 2006; 66: A83.

13- Azócar P. Sonography of the hand: tendon pathology, vascular disease, and soft tissue neoplasms. J Clin Ultrasound. 2004; 32: 47080.

14- Wang PT, Bonavita JA, DeLone FX, Jr, McClellan RM, Witham RS. Ultrasonic assistance in the diagnosis of hand flexor tendon injuries. Ann PlastSurg. 1999; 42:403407.

15- Jeyapalan K, Bisson MA, Dias JJ, Griffin $\mathbf{Y}$, Bhatt R. The role of ultrasound in the management of flexor tendon injuries. J Hand Surg Eur. 2008; 33:430-4

16- Angelides AC. Ganglions of the hand and wrist. In: Green DP, Hotchkiss RN, Pederson WC, eds. Operative Hand Surgery, 4th edn. New York: Churchill Livingstone. 1999; 2:2171-83.

17- Greendyke SD, Wilson M, Shepler TR. Anterior wrist ganglia from the scaphotrapezial joint. J Hand Surg. 1992; 17(3):487-90.

18- Middleton WD, Patel V, Teefey SA, et al. Giant cell tumors of the tendon sheath: analysis of sonographic findings. AJR Am J Roentgenol. 2004; 183: 337-339.

19- McNally EG. Ultrasound of the small joints of the hands and feet:current status. Skeletal Radiol. 2008; 37:99-113 